\title{
Common Subtype of Small Renal Mass MR Imaging Characterisation: A Medical Center Experience in Taiwan
}

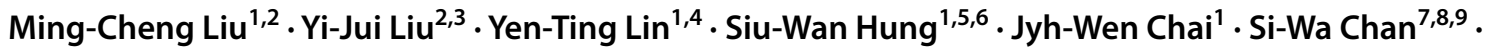 \\ Kun-Yuan Chiu ${ }^{10,11} \cdot$ Chih-Hao Chang ${ }^{12} \cdot$ Ya-Ling Tsou ${ }^{1,13}$ (1)
}

Received: 12 August 2021 / Accepted: 12 January 2022 / Published online: 3 February 2022

(C) The Author(s) 2022

\begin{abstract}
Purpose Many studies have shown that multiparametric magnetic resonance imaging (MRI) may be helpful for differentiating malignant renal cell carcinomas (RCCs) from benign lesions. However, the key imaging characteristics that differ between malignant and benign tumors still require further discussion.

Methods We analyzed 60 adult patients diagnosed with 72 small renal masses (SRMs) who received preoperative MRI from 2014 to 2019 at a hospital in Taiwan. The MRI features included conventional MRI parameters, diffusion-weighted imaging (DWI) data, and dynamic contrast-enhanced (DCE) patterns, which were documented and compared among the four common subtypes: clear cell RCC (ccRCC), papillary RCC (pRCC), angiomyolipoma (AML) and other types of RCC. The apparent diffusion coefficient (ADC) values of high- and low-grade RCCs were also analyzed.

Results The results show that ccRCC had higher T2-weighted signal intensity than the other three subgroups, a higher arterial wash-in index (AWI) and ADC value than AML and pRCC, and manifested a plateau $(n=9,25 \%)$ or washout $(\mathrm{n}=27$, $75 \%$ ) enhancement pattern. AMLs exhibited more intravoxel fat than the other three subtype groups, and half of the AMLs (6 in 12) contained bulk fat. pRCC demonstrated a more progressive $(n=3,60 \%)$ enhancement pattern than the other three subgroups. The ADC value of high-grade RCCs was significantly lower than that of low-grade RCCs.

Conclusion These findings may indicate that multiparametric MRI is useful in differentiating among four common pathological types of SRMs, and the ADC value may be helpful in evaluating the histological grade of malignancy.
\end{abstract}

Keywords Magnetic resonance imaging (MRI) $\cdot$ Apparent diffusion coefficient (ADC) $\cdot$ Renal tumor $\cdot$ Renal cell carcinoma (RCC) · Angiomyolipoma (AML)

\section{Introduction}

Small renal masses (SRMs), defined as renal tumors less than $4 \mathrm{~cm}$ in diameter, are less likely to cause clinical symptoms such as hematuria, low back pain, or palpable masses [1]. The three most common subtypes of malignant renal cell carcinoma (RCC) are clear cell RCC (ccRCC), papillary RCC (pRCC), and chromophobe RCC (chRCC) [2]. Additionally, the Fuhrman classification grading system divides RCCs into either high- or low-grade tumors based on histocytology. In clinical practice, the subtype and histopathologic grade should be taken into consideration when predicting the prognosis of RCC.

Ya-Ling Tsou

joanne19901048@gmail.com

Extended author information available on the last page of the article
In recent years, SRMs have increasingly been incidentally discovered through imaging examinations [3]. However, certain benign tumors, such as angiomyolipoma (AML) and oncocytoma, may display some similar imaging characteristics, making them difficult to differentiate from RCC [1, $4,5]$ and thus making clinicians hesitant to remove SRMs surgically or to actively surveil SRMs.

Renal tumor biopsy (RTB) can provide a pathological diagnosis for uncertain SRMs. Nevertheless, biopsy is not a reliable method for tumor grading due to the possible underestimation of a simple specimen [6]. Additionally, RTB is an invasive procedure with possible complications, such as hemorrhage, infection, pneumothorax, and hollow organ perforation. In the diagnosis or selection of treatment for these SRMs, novel imaging modalities are still useful first tools [7]. 
Computed tomography (CT) and magnetic resonance imaging (MRI) are the first-line noninvasive imaging methods for both the diagnosis and staging of SRMs. MRI is considered a better tool than CT because there is no radiation exposure to the patient, and diffusion images can also provide extra information in patients with poor renal function for whom contrast medium administration is unsuitable [8]. Multiple MRI parameters can show the intratumoral content of SRMs. For example, T2-weighted, T1-weighted, and chemical shift imaging can reveal manifestations such as hemorrhage, protein, macroscopic fat, or microscopic fat within the tumor; the postcontrast series of dynamic contrast-enhanced (DCE) images can demonstrate the enhancement pattern of solid tumors, while diffusion-weighted imaging (DWI) with apparent diffusion coefficient (ADC) values can illustrate the cellular microenvironment of the tumor by assessing the movement restrictions of free water molecules. These imaging features may be useful in differentiating malignant RCC from benign lesions and classifying RCC subtypes and histological grades [1].

Multiparametric MRI has been reported to be helpful in differentiating malignant RCCs from benign lesions in several research studies [9-12]. However, summaries of the diagnostic values of such parameters have seldom been reported. Therefore, we focused on four pathological subtypes (ccRCC, pRCC, AML, and other types of RCC) and analyzed the MRI sequences reported to be useful in diagnosing high- and low-grade RCCs as well as benign renal tumors in previous studies.

\section{Methods}

\subsection{Subjects}

This retrospective study was approved by our Institutional Review Board. By reviewing the medical records from a medical center in Taiwan, this retrospective study identified patients who had undergone surgical resection of a renal mass measuring less than $4 \mathrm{~cm}$ from 2014 to 2019 who also had preoperative multiparametric MRI data of the kidney available for analysis. Patients with renal masses larger than $4 \mathrm{~cm}$ who had a pathologic diagnosis of urothelial carcinoma or renal metastasis were not included. Sixty-eight patients with a total of 80 SRMs were initially selected. Six patients with benign renal cysts and two with benign inflammatory disease were excluded because our study aimed to analyze tumor masses.

Finally, 60 adult patients with a total of 72 SRMs were included: 58 (80.6\%) with pathologically confirmed RCC, including 38 (52.8\%) ccRCCs, 9 (12.5\%) pRCCs, 3 (4.2\%) chromophobe RCCs (chRCCs), and 8 (11.1\%) other RCC types (4 tuberous sclerosis-associated RCCs, 1 unclassified
RCC, 1 Xp11 translocation RCC, and 2 acquired cystic disease-associated RCCs). Additionally, there were 14 SRMs (19.4\%) considered benign renal tumors: 12 (16.7\%) AMLs (1 AML with an epithelioid component) and 2 (2.8\%) oncocytomas. Due to the small number of chRCC $(n=3)$ and oncocytoma $(n=2)$ patients, we did not include these two tumor types in our tumor subtype analysis. We divided the SRMs into four subgroups for comparisons: ccRCC, pRCC, other types of RCC, and AML.

\subsection{Image Acquisition}

All patients with SRMs underwent MRI using both conventional MRI and DWI sequences. DCE MRI was administered if the patient's renal function allowed. MRI was performed with a 1.5-T body scanner (MAGNETOM AERA, Siemens, Erlangen, Germany) using an 18-channel phased-array body coil with the following protocols:

(1) Axial T2-weighted half-Fourier acquisition single-shot turbo spin-echo (HASTE): repetition time $(\mathrm{TR})=1200 \mathrm{~ms}$, echo time $(\mathrm{TE})=95 \mathrm{~ms}$, flip angle $=160^{\circ}$, field of view $=36 \times 30 \mathrm{~cm}$, matrix $=320 \times 182$, slice thickness $=5 \mathrm{~mm}$, and gap $=0 \mathrm{~mm}$.

(2) Axial T1-weighted fast spoiled gradient-recalled echo dual-echo (FSPGR-DE): TR $=142 \mathrm{~ms}$, $\mathrm{TE}=2.38 \mathrm{~ms}$ and $4.76 \mathrm{~ms}$, flip angle $=70^{\circ}$, field of view $=36 \times 30 \mathrm{~cm}$, and matrix $=256 \times 166$.

(3) Axial DWI: TR $=3300 \mathrm{~ms}$, $\mathrm{TE}=65 \mathrm{~ms}$, field of view $=38 \times 31 \mathrm{~cm}$, matrix $=192 \times 125$, number of excitations $(\mathrm{NEX})=3$, bandwidth $=1736 \mathrm{~Hz} /$ pixel, diffusion mode $=3$-scan trace, slice thickness $=5 \mathrm{~mm}$, and gap $=0 \mathrm{~mm}$ with $\mathrm{b}$ values $=0$ and $800 \mathrm{~s} / \mathrm{mm}^{2}$ [13-15]. DWI was performed by using a single-shot spin-echo echo-planar imaging sequence with a parallel imaging technique and fat saturation.

(4) Dynamic MRI was performed in multiple breath-holds (BHs) using axial 3D fat-suppressed T1-weighted volumetric interpolated $\mathrm{BH}$ (VIBE) sequences: $\mathrm{TR}=4.5 \mathrm{~ms}, \mathrm{TE}=2.2 \mathrm{~ms}$, flip angle $=15^{\circ}$, field of view $=38 \times 38 \mathrm{~cm}$, matrix $=320 \times 192$, slice thickness $=5 \mathrm{~mm}$, and gap $=0 \mathrm{~mm}$.

A gadolinium-based contrast medium was used for the dynamic scan at a dose of $0.1 \mathrm{mmol} / \mathrm{kg}$ of body weight as a bolus injection. Corticomedullary and late nephrographic phase images were acquired 30 and $90 \mathrm{~s}$ after the contrast medium injection, respectively.

These image parameters remained constant from 2014 to 2019 ; only the set of $b$ values changed from four $b$ values to seven $b$ values (both sets including $b$ values of 0 and $800 \mathrm{~s} /$ $\mathrm{mm}^{2}$ ), and this had no effect on the ADC calculation. 


\subsection{Image Interpretation and Definition of Features}

The data of different MRI sequences, including the T2-weighted image signal intensity, intravoxel fat, bulk fat, DCE enhancement pattern, AWI, and ADC values, were recorded. There were no postprocessing changes to image signal intensity in this study. The definition of each MRI feature with its respective grading scale is summarized in Table 1 . These images were reviewed and measured by a genitourinary radiologist with five years of experience. Qualitative results were determined by careful visual comparison by a professional genitourinary radiologist; quantitative results, such as the AWI and ADC, were calculated through ratios. A region of interest (ROI) was placed within a portion of the solid area where the minimum ADC value was identified through visual inspection of the ADC map. The necrotic and hemorrhagic regions were identified on conventional MRI scans and were avoided.

\subsection{Statistical Analysis}

First, we used the Kolmogorov-Smirnov test to evaluate the normality of the continuous variables, including age, tumor size, arterial wash-in index (AWI), and ADC value. These samples were standardized and compared with a standard normal distribution to determine whether the parametric or nonparametric method should be applied for data analysis. One-way ANOVA (parametric method) and the Kruskal-Wallis test (nonparametric method) were performed to compare the parameters of age, tumor size, AWI, and ADC value among the different renal tumor subtypes. A Dunn-Bonferroni post hoc test was also applied to further select pairwise comparisons. The $\chi^{2}$ test was used for the comparison of categorical variables, such as conventional MRI features, sex, and enhancement patterns.

The t-test (parametric method) and Mann-Whitney U test (nonparametric method) were applied to compare the parameters of AWI and ADC values between low-grade and high-grade malignant tumors. A box-and-whisker plot was created to compare the AWI and ADC values of benign renal lesions and RCCs of different histological subtypes.

SPSS 22.0 software was used for data analysis. Both the $\mathrm{AWI}$ and $\mathrm{ADC}$ values are expressed as the mean $\pm \mathrm{SD}$. The results were considered statistically significant when the $p$ value was $<0.05$.

\section{Results}

\subsection{Comparisons Among Different Tumor Subtypes}

We compared the imaging and basic features of the four subtypes of renal tumors, ccRCC, AML, pRCC, and other types of RCC. There were significant differences among the four groups of tumors in terms of age, AWI, ADC, size, T2-weighted signal intensity, intravoxel fat, bulk fat, and enhancement pattern $(p=0.033,<0.001,<0.001,0.012,<$ $0.001,<0.001,<0.001$, and $<0.001$, respectively). There were no significant differences among these four subtypes regarding sex $(p=0.056)$ and grade $(p=0.426)$. Finally, the pairwise comparisons that showed the significant differences in the above parameters among the groups of tumors are listed in Tables 2 and 3.

In the comparison of ccRCC and AML, ccRCC exhibited a higher T2-weighted signal intensity (high: $81.6 \%$ vs.

Table 1 Definition of the imaging features and their grading scales

\begin{tabular}{|c|c|c|}
\hline Feature & Definition & Categories \\
\hline \multicolumn{3}{|l|}{ Conventional MRI } \\
\hline T2W SI & $\begin{array}{l}\text { Intensity of the lesion in comparison with the intensity of the normal renal cortex } \\
\text { on non-fat-suppressed T2W images }\end{array}$ & $\begin{array}{l}\text { 1: Low } \\
\text { 2: Isointense } \\
\text { 3: High }\end{array}$ \\
\hline Intravoxel fat (microscopic fat) & Signal dropout on opposed-phase images compared with that on in-phase images & $\begin{array}{l}\text { 1: Absent } \\
\text { 2: Present }\end{array}$ \\
\hline Bulk fat (macroscopic fat) & $\begin{array}{l}\text { High signal intensity on non-fat-suppressed } \mathrm{T} 2 \mathrm{~W} \text { images, with signal dropout on } \\
\text { fat-suppressed images }\end{array}$ & $\begin{array}{l}\text { 1: Absent } \\
\text { 2: Present }\end{array}$ \\
\hline \multicolumn{3}{|l|}{ DCE MRI } \\
\hline Enhancement pattern & $\begin{array}{l}\text { Mass enhancement during the late nephrographic phase, as follows: } \\
\text { - Progressive: at least } 10 \% \text { more than that in the corticomedullary phase } \\
\text { - Plateau: approximately } 10 \% \text { of that the corticomedullary phase } \\
\text { - Washout: at least } 10 \% \text { less than that in the corticomedullary phase }\end{array}$ & $\begin{array}{l}\text { 1: Progressive } \\
\text { 2: Plateau } \\
\text { 3: Washout }\end{array}$ \\
\hline Arterial wash-in index & Arterial wash-in index (postcontrast arterial phase) & $\left.\left[\mathrm{SI}_{\mathrm{a}}-\mathrm{SI}_{\mathrm{pre}}\right] / \mathrm{SI}_{\mathrm{pre}} \times 100\right)$ \\
\hline DWI MRI & $\mathrm{b}$ values for $\mathrm{ADC}\left(\mathrm{s} / \mathrm{mm}^{2}\right)=0,800$ & ADC level $\left(\times 10^{-3} \mathrm{~mm}^{2} / \mathrm{s}\right)$ \\
\hline
\end{tabular}

T2W SI T2-weighted signal intensity, SI signal intensity 
Table 2 Comparison of different MRI parameters between each group of tumors (continuous variables)

\begin{tabular}{|c|c|c|c|c|c|c|c|c|c|}
\hline Group & & $\mathrm{n}$ & Mean & $\pm \mathrm{SD}$ & $p$ value & \multicolumn{4}{|c|}{ Dunn-Bonferroni test } \\
\hline 1 & ccRCC & 38 & 50.5 & \pm 16.5 & & 1 vs. 2 & 1.000 & 2 vs. 3 & 1.000 \\
\hline 2 & AML & 12 & 56.9 & \pm 14.3 & & 1 vs. 3 & 0.997 & 2 vs. 4 & 0.060 \\
\hline 3 & $\mathrm{pRCC}$ & 9 & 59.1 & \pm 7.3 & & 1 vs. 4 & 0.368 & 3 vs. 4 & 0.064 \\
\hline \multirow[t]{2}{*}{4} & Other type RCC & 8 & 40.5 & \pm 6.0 & & & & & \\
\hline & AWI & & & & $<0.001 * *$ & & & & \\
\hline 1 & $\mathrm{ccRCC}$ & 36 & 260.9 & \pm 116.7 & & 1 vs. 2 & $0.015^{*}$ & 2 vs. 3 & 0.453 \\
\hline 2 & AML & 12 & 165.4 & \pm 56.5 & & 1 vs. 3 & $<0.001 * *$ & 2 vs. 4 & 0.436 \\
\hline 3 & $\mathrm{pRCC}$ & 5 & 30.5 & \pm 13.7 & & 1 vs. 4 & 1.000 & 3 vs. 4 & $0.014^{*}$ \\
\hline \multirow[t]{2}{*}{4} & Other type RCC & 6 & 225.2 & \pm 49.8 & & & & & \\
\hline & $\mathrm{ADC} \times 10^{-3} \mathrm{~mm}^{2} / \mathrm{s}$ & & & & $<0.001 * *$ & & & & \\
\hline 1 & $\operatorname{ccRCC}$ & 38 & 1.907 & \pm 0.336 & & 1 vs. 2 & $<0.001 * *$ & 2 vs. 3 & 1.000 \\
\hline 2 & AML & 12 & 1.191 & \pm 0.180 & & 1 vs. 3 & $<0.001 * *$ & 2 vs. 4 & 0.423 \\
\hline 3 & $\mathrm{pRCC}$ & 9 & 0.986 & \pm 0.202 & & 1 vs. 4 & 0.223 & 3 vs. 4 & 0.094 \\
\hline \multirow[t]{2}{*}{4} & Other type RCC & 8 & 1.524 & \pm 0.255 & & & & & \\
\hline & Size & & & & $0.012 *$ & & & & \\
\hline 1 & $\mathrm{ccRCC}$ & 38 & 2.8 & \pm 1.0 & & 1 vs. 2 & 1.000 & 2 vs. 3 & 1.000 \\
\hline 2 & AML & 12 & 2.6 & \pm 1.0 & & 1 vs. 3 & 0.594 & 2 vs. 4 & 0.111 \\
\hline 3 & $\mathrm{pRCC}$ & 9 & 2.1 & \pm 1.0 & & 1 vs. 4 & $0.012 *$ & 3 vs. 4 & 1.000 \\
\hline 4 & Other type RCC & 8 & 1.5 & \pm 0.6 & & & & & \\
\hline
\end{tabular}

$* p<0.05, * * p<0.01$

Table 3 Comparison of different MRI parameters between each group of tumors (categorical variables)

\begin{tabular}{|c|c|c|c|c|c|c|c|c|c|}
\hline \multirow[t]{2}{*}{ Group } & & & & & $p$ value & \multicolumn{4}{|c|}{$\chi^{2}$ Post hoc } \\
\hline & & & & & & $p$ value & & & \\
\hline & T2W SI & Low & Isointense & High & $<0.001 * *$ & & & & \\
\hline 1 & $\operatorname{ccRCC}(\mathrm{n}=38)$ & $2(5.3 \%)$ & $5(13.2 \%)$ & $31(81.6 \%)$ & & 1 vs. 2 & $<0.001 * *$ & 2 vs. 3 & 0.621 \\
\hline 2 & $\operatorname{AML}(\mathrm{n}=12)$ & $8(66.7 \%)$ & $2(16.7 \%)$ & $2(16.7 \%)$ & & 1 vs. 3 & $<0.001 * *$ & 2 vs. 4 & 0.578 \\
\hline 3 & $\operatorname{pRCC}(n=9)$ & $7(77.8 \%)$ & $0(0.0 \%)$ & $2(22.2 \%)$ & & 1 vs. 4 & $0.026^{*}$ & 3 vs. 4 & 0.308 \\
\hline \multirow[t]{2}{*}{4} & Other type RCC $(\mathrm{n}=8)$ & $3(37.5 \%)$ & $2(25.0 \%)$ & $3(37.5 \%)$ & & & & & \\
\hline & Intravoxel fat & Absent & Present & & $<0.001 * *$ & & & & \\
\hline 1 & $\operatorname{ccRCC}(\mathrm{n}=38)$ & $34(89.5 \%)$ & $4(10.5 \%)$ & & & 1 vs. 2 & $<0.001 * *$ & 2 vs. 3 & $0.003 * *$ \\
\hline 2 & $\operatorname{AML}(\mathrm{n}=12)$ & $3(25.0 \%)$ & $9(75.0 \%)$ & & & 1 vs. 3 & 0.862 & 2 vs. 4 & $0.003 * *$ \\
\hline 3 & $\mathrm{pRCC}(\mathrm{n}=9)$ & $9(100.0 \%)$ & $0(0.0 \%)$ & & & 1 vs. 4 & 1.000 & 3 vs. 4 & 1.000 \\
\hline \multirow[t]{2}{*}{4} & Other type RCC $(\mathrm{n}=8)$ & $8(100.0 \%)$ & $0(0.0 \%)$ & & & & & & \\
\hline & Bulk fat & Absent & Present & & $<0.001 * *$ & & & & \\
\hline 1 & $\operatorname{ccRCC}(\mathrm{n}=38)$ & $38(100.0 \%)$ & $0(0.0 \%)$ & & & 1 vs. 2 & $<0.001 * *$ & 2 vs. 3 & 0.056 \\
\hline 2 & $\operatorname{AML}(\mathrm{n}=12)$ & $6(50.0 \%)$ & $6(50.0 \%)$ & & & 1 vs. 3 & 1.000 & 2 vs. 4 & 0.084 \\
\hline 3 & $\mathrm{pRCC}(\mathrm{n}=9)$ & $9(100.0 \%)$ & $0(0.0 \%)$ & & & 1 vs. 4 & 1.000 & 3 vs. 4 & 1.000 \\
\hline \multirow[t]{2}{*}{4} & Other type RCC $(\mathrm{n}=8)$ & $8(100.0 \%)$ & $0(0.0 \%)$ & & & & & & \\
\hline & Enhancement pattern & Progressive & Plateau & Washout & $<0.001 * *$ & & & & \\
\hline 1 & $\operatorname{ccRCC}(\mathrm{n}=36)$ & $0(0.0 \%)$ & $9(25.0 \%)$ & $27(75.0 \%)$ & & 1 vs. 2 & $0.023^{*}$ & 2 vs. 3 & $0.010^{*}$ \\
\hline 2 & $\operatorname{AML}(\mathrm{n}=12)$ & $2(16.7 \%)$ & $0(0.0 \%)$ & $10(83.3 \%)$ & & 1 vs. 3 & $<0.001 * *$ & 2 vs. 4 & 0.488 \\
\hline 3 & $\mathrm{pRCC}(\mathrm{n}=5)$ & $3(60.0 \%)$ & $2(40.0 \%)$ & $0(0.0 \%)$ & & 1 vs. 4 & 1.000 & 3 vs. 4 & $0.023^{*}$ \\
\hline 4 & Other type RCC $(n=6)$ & $0(0.0 \%)$ & $1(16.7 \%)$ & $5(83.3 \%)$ & & & & & \\
\hline
\end{tabular}

$* p<0.05, * * p<0.01$ 
$16.7 \%$, and low: $5.3 \%$ vs. $66.7 \%)(p<0.001)$; less intravoxel fat (present: $10.5 \%$ vs. $75.0 \%$, and absent: $89.5 \%$ vs. $25.0 \%, p<0.001$ ); less bulk fat (present: $0.0 \%$ vs. $50.0 \%$, and absent: $100.0 \%$ vs. $50.0 \%, p<0.001$ ); more type 2 and 3 enhancement patterns (types 2 and $3: 100.0 \%$ vs. $83.3 \%$, $p=0.023)$; a higher AWI $(260.9 \pm 116.7$ vs. $165.4 \pm 56.5$, $p=0.015)$; and a higher ADC value $(1.907 \pm 0.336 \mathrm{vs}$. $1.191 \pm 0.180, p<0.001)$.

In the comparison of ccRCC and pRCC, ccRCC exhibited a higher T2-weighted signal intensity (high: $81.6 \%$ vs. $22.2 \%$, and low: $5.3 \%$ vs. $77.8 \%, p<0.001$ ); more type 3 enhancement patterns (type $3: 75.0 \%$ vs. $0.0 \%$, and type 1 : $0.0 \%$ vs. $60.0 \%, p<0.001)$; a higher AWI $(260.9 \pm 116.7$ vs. $30.5 \pm 13.7, p<0.001)$; and a higher ADC value $(1.907 \pm 0.336$ vs. $0.986 \pm 0.202, p<0.001)$.

In the comparison of ccRCC and other types of RCC, ccRCC exhibited a higher T2-weighted signal intensity (high: $81.6 \%$ vs. $37.5 \%$, and low: $5.3 \%$ vs. $37.5 \%, p=0.026$ ) and larger size $(2.8 \pm 1.0$ vs. $1.5 \pm 0.6, p=0.012)$.

In the comparison of AML and pRCC, AML exhibited more intravoxel fat (present: $75.0 \%$ vs. $0.0 \%$, and absent: $25.0 \%$ vs. $100.0 \%, p=0.003$ ) and more type 3 enhancement patterns (type $3: 83.3 \%$ vs. $0.0 \%$, and type $1: 16.7 \%$ vs. $60.0 \%, p=0.010$ ).

In the comparison of AML and other types of RCC, AML exhibited more intravoxel fat (present: $75.0 \%$ vs. $0.0 \%$, and absent: $25.0 \%$ vs. $100.0 \%, p=0.003$ ).

In the comparison of pRCC and other types of RCC, pRCC exhibited more type 1 enhancement patterns (type 3 : $0.0 \%$ vs. $83.3 \%$, and type $1: 60.0 \%$ vs. $0.0 \%, p=0.023)$ and a lower AWI (30.5 \pm 13.7 vs. $225.2 \pm 49.8, p=0.014)$.

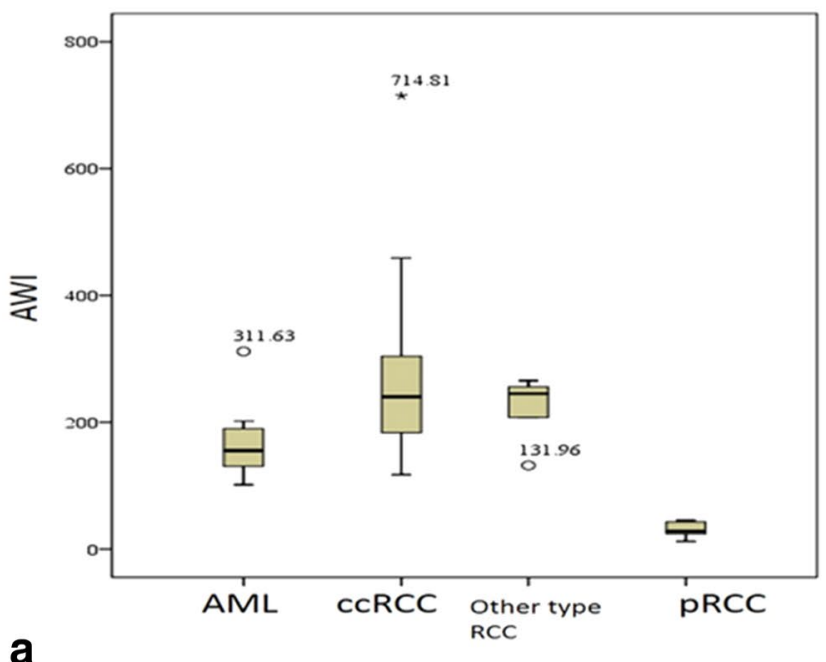

Fig. 1 a Box-and-whisker plot of the AWIs of SRMs, and b boxand-whisker plot of the ADC values of SRMs [the boxes indicate the interquartile range, the whiskers indicate the range of all values, and
Eight patients (12\%) with poor renal function did not undergo contrast-enhanced imaging, limiting the data collected for determination of the AWI and DCE values. However, since these MRI parameters are independent factors, these missing values did not affect the remaining parameters.

Box-and-whisker plots of the AWI and ADC values of ccRCC, pRCC, other types of RCC, and AML are shown in Fig. 1.

Figures 2, 3 and 4 demonstrate the typical image features of the major types of SRMs. Figure 2 illustrates the MRI images of a Fuhrman grade II ccRCC. The images show that the T2-weighted signal intensity of the tumor is higher than that of the normal renal cortex. There is no obvious signal dropout on the fat-suppressed images compared with nonfatsuppressed T2-weighted images, which indicates that there is no detectable bulk fat. Additionally, there is no obvious signal dropout on the opposed-phase images compared with in-phase images, which indicates that there is no detectable intravoxel fat; the AWI is 244.16, with the presence of a plateau pattern and an ADC value $=1.911 \times 10^{-3} \mathrm{~mm}^{2} / \mathrm{s}$.

Figure 3 illustrates the MRI images of a benign AML. The images show that the T2-weighted signal intensity of the tumor is lower than that of the normal renal cortex. There is a detectable signal dropout on the opposed-phase images compared with that on the in-phase images, which indicates the presence of intravoxel fat. The AWI was 150.00, with the presence of a washout enhancement pattern and an ADC value $=1.201 \times 10^{-3} \mathrm{~mm}^{2} / \mathrm{s}$.

Figure 4 illustrates the MRI images of a Fuhrman grade III pRCC. The images show that the T2-weighted signal intensity of the tumor is lower than that of the normal renal cortex. There was no bulk or intravoxel fat component detected

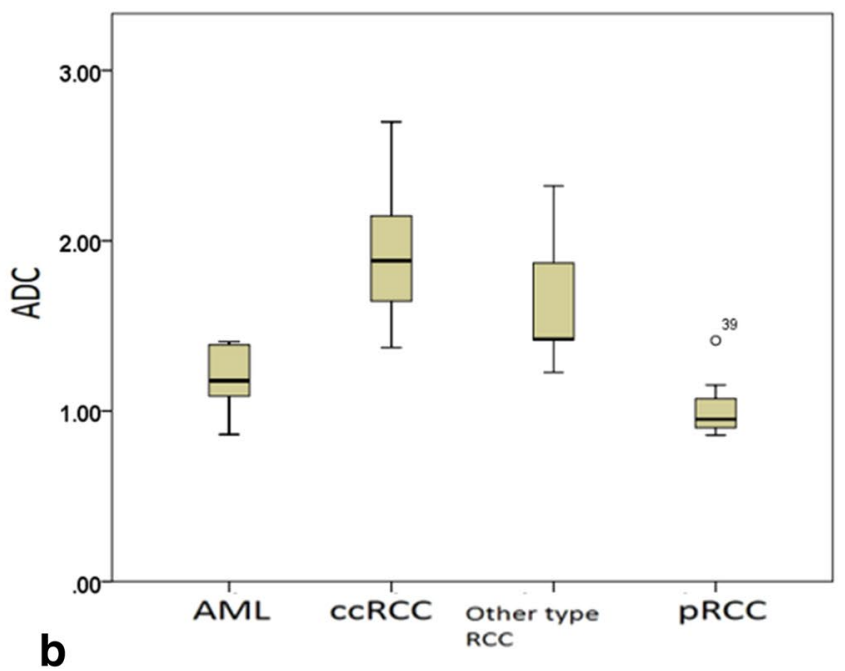

the horizontal line within the box indicates the median value, confidence interval (CI)-95\%] 


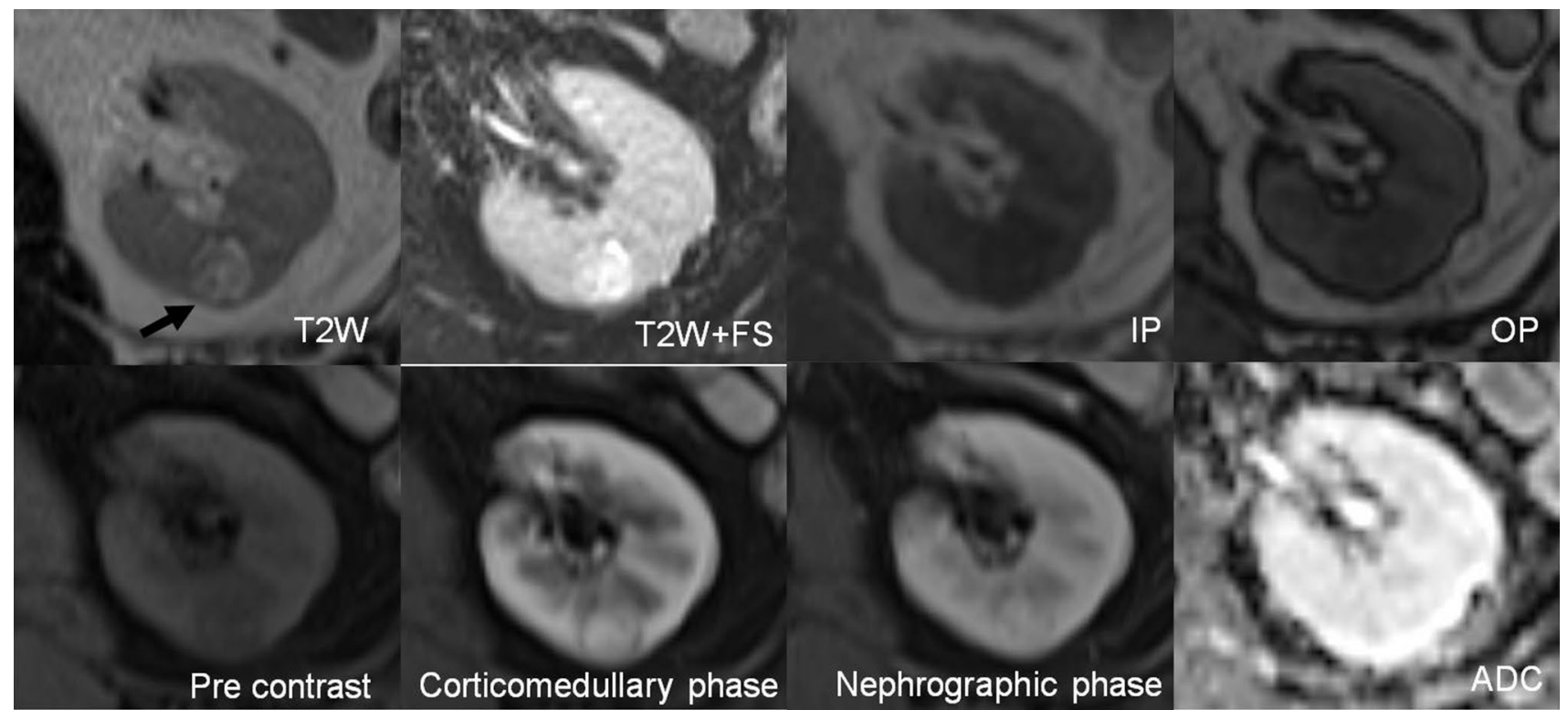

Fig. 2 MRI scans of ccRCC. Note T2W T2-weighted signal intensity, $F S$ fat-suppressed, $I P$ in-phase images, $O P$ opposed-phase images, $A D C$ apparent diffusion coefficient

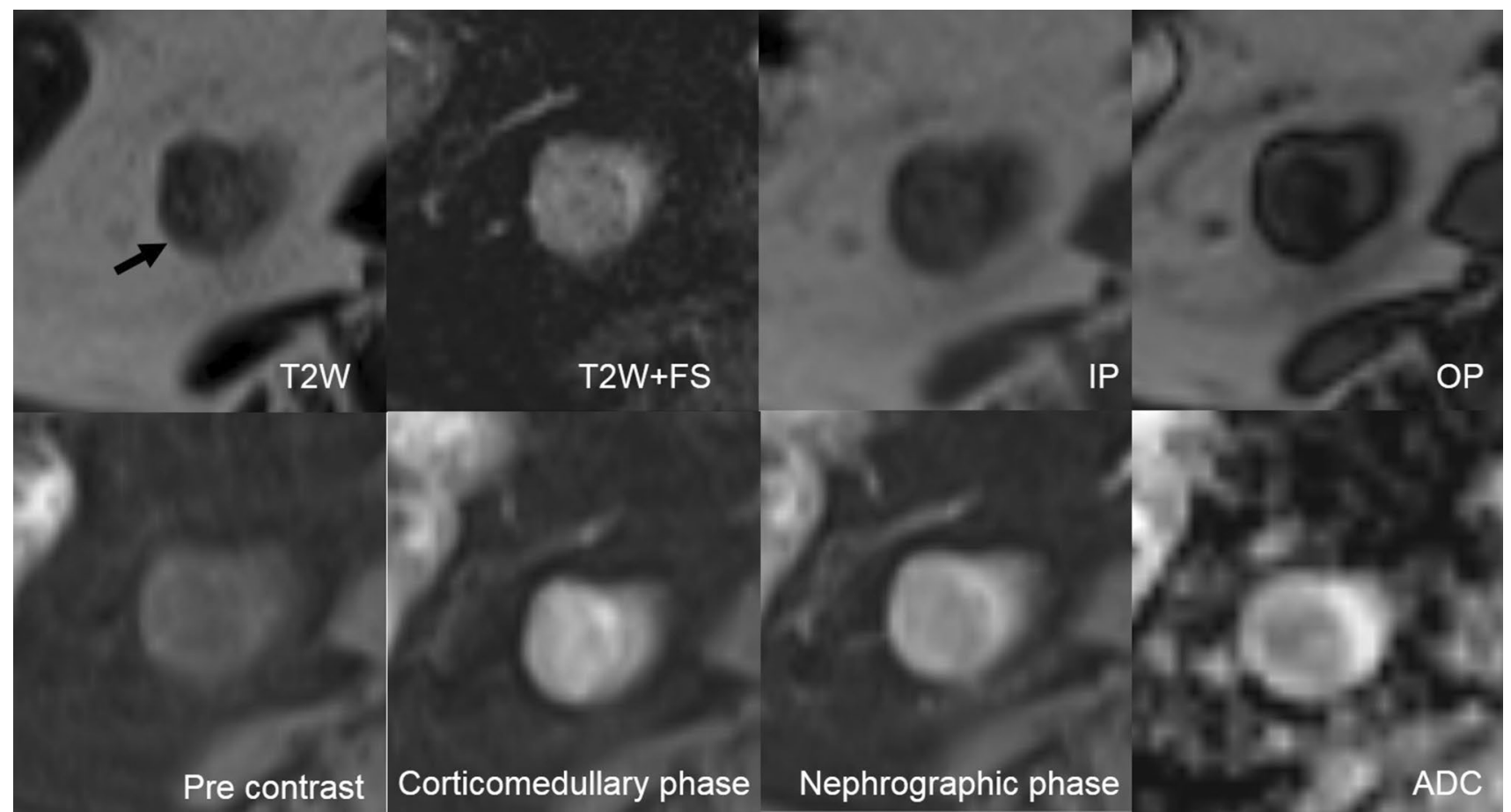

Fig. 3 MRI scans of AML. Note T2W T2-weighted signal intensity, FS fat-suppressed, $I P$ in-phase images, $O P$ opposed-phase images, $A D C$ apparent diffusion coefficient

in fat-suppressed images or opposed-phase sequences. The AWI was 28.10, with the presence of a progressive enhancement pattern and an ADC value $=0.896 \times 10^{-3} \mathrm{~mm}^{2} / \mathrm{s}$.

\subsection{Tumor Grade Comparison}

The study also analyzed differences in imaging and basic features between low-grade and high-grade RCCs, and these results are shown in Table 4. Compared with patients 


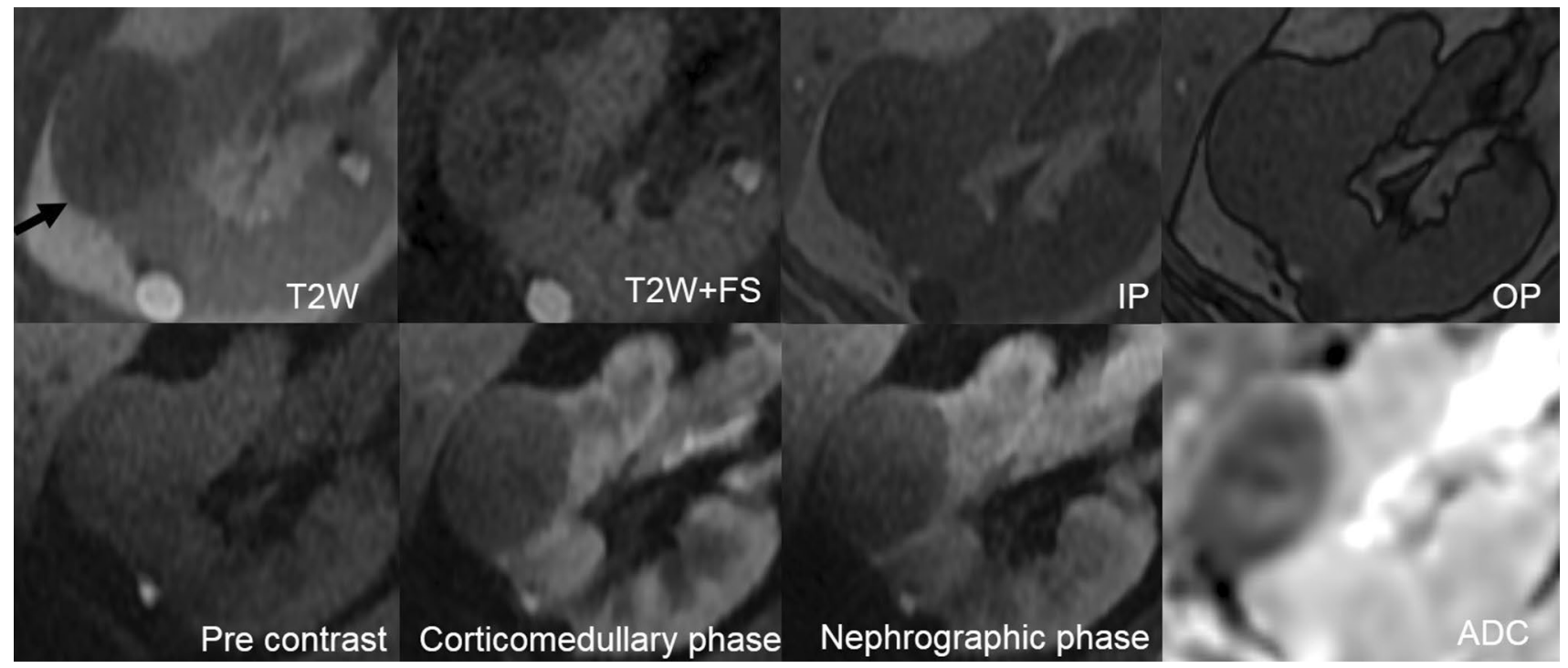

Fig. 4 MRI scans of pRCC. Note T2W T2-weighted signal intensity, FS fat-suppressed, $I P$ in-phase images, $O P$ opposed-phase images, $A D C$ apparent diffusion coefficient

Table 4 Comparison of imaging and basic features between low-grade and high-grade ccRCCs and among all subtypes of RCCs

\begin{tabular}{|c|c|c|c|c|c|c|c|c|c|c|c|}
\hline & & \multicolumn{2}{|c|}{$\begin{array}{l}\text { LG-ccRCC } \\
(\mathrm{n}=32)\end{array}$} & \multicolumn{2}{|c|}{$\begin{array}{l}\text { HG-ccRCC } \\
(n=6)\end{array}$} & \multirow[t]{2}{*}{$p$ value } & \multicolumn{2}{|c|}{$\begin{array}{l}\text { LG-all RCCs } \\
(\mathrm{n}=48)\end{array}$} & \multicolumn{2}{|c|}{$\begin{array}{l}\text { HG-all RCCs } \\
(\mathrm{n}=10)\end{array}$} & \multirow[t]{2}{*}{$p$ value } \\
\hline & & $\mathrm{n}$ & $\%$ & $\mathrm{n}$ & $\%$ & & $\mathrm{n}$ & $\%$ & $\mathrm{n}$ & $\%$ & \\
\hline \multicolumn{2}{|l|}{ Patient age $($ mean $\pm S D)$} & 47.9 & \pm 16.4 & 64.7 & \pm 8.6 & $0.024 *$ & 49.1 & \pm 15.4 & 61.7 & \pm 10.0 & $0.015^{*}$ \\
\hline \multirow[t]{2}{*}{$\operatorname{Sex}{ }^{\mathrm{f}}$} & Male & 20 & $(62.5 \%)$ & 5 & $(83.3 \%)$ & 0.643 & 30 & $(62.5 \%)$ & 9 & $(90.0 \%)$ & \multirow[t]{2}{*}{0.142} \\
\hline & Female & 12 & $(37.5 \%)$ & 1 & $(16.7 \%)$ & & 18 & $(37.5 \%)$ & 1 & $(10.0 \%)$ & \\
\hline AWI $($ mean $\pm S D)(n=36)$ & & 273.2 & \pm 119.2 & 185.0 & \pm 64.3 & 0.066 & 239.6 & \pm 128.6 & 155.0 & \pm 90.1 & 0.067 \\
\hline $\mathrm{ADC}\left(\times 10^{-3} \mathrm{~mm}^{2} / \mathrm{s}\right.$, mean $\left.\pm \mathrm{SD}\right)$ & & 1.986 & \pm 0.302 & 1.485 & \pm 0.118 & $<0.001 * *$ & 1.764 & \pm 0.445 & 1.299 & \pm 0.281 & $0.001 * *$ \\
\hline Size $($ mean $\pm S D)$ & & 2.7 & \pm 1.0 & 3.4 & \pm 0.9 & 0.090 & 2.4 & \pm 1.0 & 3.0 & \pm 1.0 & 0.101 \\
\hline \multirow[t]{3}{*}{ T2W SI } & 1 & 1 & $(3.1 \%)$ & 1 & $(16.7 \%)$ & \multirow[t]{3}{*}{0.089} & 8 & $(16.7 \%)$ & 4 & $(40.0 \%)$ & \multirow[t]{3}{*}{0.070} \\
\hline & 2 & 3 & $(9.4 \%)$ & 2 & $(33.3 \%)$ & & 7 & $(14.6 \%)$ & 3 & $(30.0 \%)$ & \\
\hline & 3 & 28 & $(87.5 \%)$ & 3 & $(50.0 \%)$ & & 33 & $(68.8 \%)$ & 3 & $(30.0 \%)$ & \\
\hline \multirow[t]{2}{*}{ Intravoxel fat } & 1 & 28 & $(87.5 \%)$ & 6 & $(100.0 \%)$ & \multirow[t]{2}{*}{1.000} & 44 & $(91.7 \%)$ & 10 & $(100.0 \%)$ & \multirow[t]{2}{*}{1.000} \\
\hline & 2 & 4 & $(12.5 \%)$ & 0 & $(0.0 \%)$ & & 4 & $(8.3 \%)$ & 0 & $(0.0 \%)$ & \\
\hline Enhancement & 1 & 0 & $(0.0 \%)$ & 0 & $(0.0 \%)$ & \multirow[t]{3}{*}{0.302} & 3 & $(7.3 \%)$ & 1 & $(12.5 \%)$ & \multirow[t]{3}{*}{0.586} \\
\hline \multirow[t]{2}{*}{ Pattern $(\mathrm{n}=36)^{\mathrm{f}}$} & 2 & 9 & $(29.0 \%)$ & 0 & $(0.0 \%)$ & & 12 & $(29.3 \%)$ & 1 & $(12.5 \%)$ & \\
\hline & 3 & 22 & $(71.0 \%)$ & 5 & $(100.0 \%)$ & & 26 & $(63.4 \%)$ & 6 & $(75.0 \%)$ & \\
\hline
\end{tabular}

$L G$ low-grade, $H G$ high-grade, $\chi^{2}$ test

$* p<0.05, * * p<0.01$

${ }^{\mathrm{f}}$ Fisher's exact test. Mann-Whitney U test

with low-grade ccRCCs, those with high-grade ccRCCs were significantly older $(p=0.024)$, and the ADC value was lower for high-grade ccRCCs $(p<0.001)$. Compared with patients with any subtype of RCC (ccRCC, pRCC, chRCC, and other type RCCs) considered low grade, those with high-grade RCCs were also older $(p=0.015)$, and the ADC value was lower for high-grade RCCs $(p=0.001)$.
There were no significant differences between low-grade and high-grade ccRCCs or among all subtypes of RCCs in the parameters of sex, AWI, size, T2-weighted signal intensity, intravoxel fat or enhancement pattern.

The box-and-whisker plot of ADC values between lowgrade and high-grade ccRCCs and the corresponding data for all subtypes of RCC are shown in Fig. 5. 


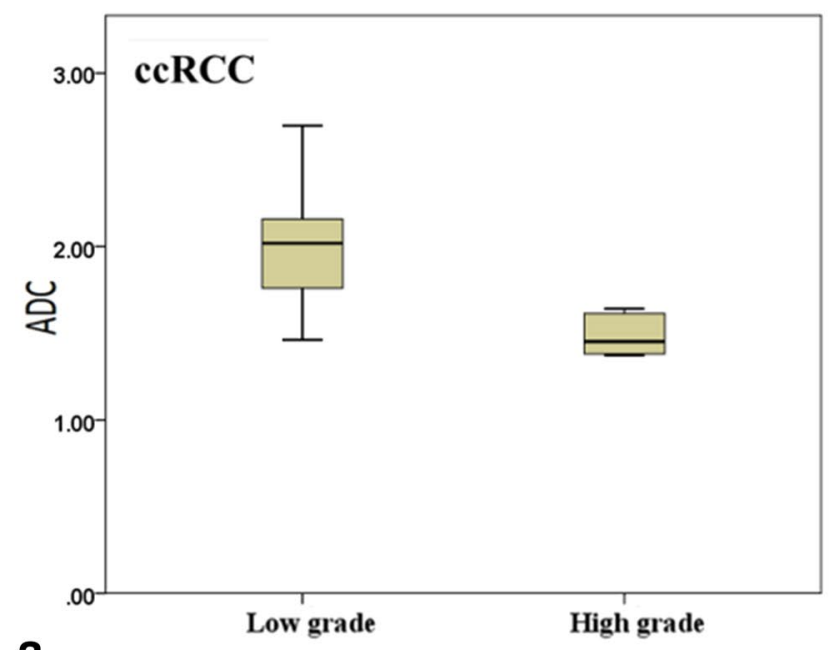

a

Fig. 5 a Box-and-whisker plot of the ADC values of low-grade and high-grade ccRCCs, and $\mathbf{b}$ box-and-whisker plot of the ADC values of low-grade and high-grade RCCs of any subtype [the boxes indicate

\section{Discussion}

In our study, we found that ccRCC showed a higher T2-weighted signal intensity than AML, pRCC, and other types of RCC. This result is in agreement with those from both Kay et al. [3] and Roy et al. [16], suggesting that pRCC typically has a low signal intensity in T2-weighted images and that ccRCC mostly displays a higher signal intensity on T2-weighted images than pRCC, chRCC, and lipid-poor AML.

In essence, AMLs are benign tumors, and regular follow-up imaging is recommended over surgical resection. However, all of the AMLs in our study were pathologically diagnosed through surgery because these were all lipid-poor AMLs that were difficult to distinguish from malignant tumors simply through imaging findings. In our study, bulk fat was found only in AMLs (6 in 12 AMLs), while intravoxel fat was found in both AMLs (9 in 12) and ccRCCs (4 in 34). These findings indicate that the presence of microscopic or macroscopic fat is still the key factor in differentiating AMLs from RCCs. A previous study found that the presence of intravoxel fat may indicate that the tumor is ccRCC rather than another subtype of RCC [17]. Consistently, none of the other subtypes of RCC were found to have an intratumoral microscopic fat component in our study.

On contrast-enhanced images, the AWI is a quantitative parameter, and the DCE pattern is a qualitative parameter. In our research, ccRCC exhibited a higher AWI than both AML and pRCC, which is in agreement with the findings of Sun et al. [2] and Kay et al. [3] in that there is higher contrast enhancement in ccRCCs than in pRCCs. chRCC had an intermediate signal intensity between that of ccRCC

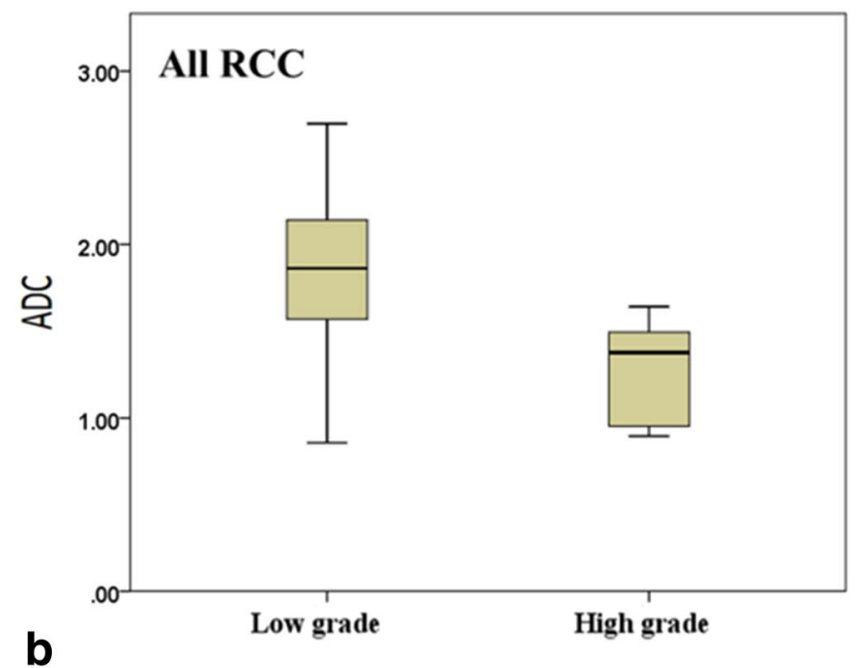

the interquartile range, the whiskers indicate the range of all values, and the horizontal line within the box indicates the median value, confidence interval (CI)—95\%]

and pRCC. Cornelis et al. [18] also showed that the AWI tended to be lower for pRCCs than for lipid-poor AMLs; however, our study did not observe statistically significant differences between these two tumors. All ccRCCs in our study exhibited a plateau- or washout-type enhancement pattern. These results indicate that the enhancement pattern can play some type of role in differentiating among these tumors. However, this is in contrast to Kay et al. [3] who found nonsignificant differences in enhancement pattern among these tumors. Therefore, further studies on the diagnostic value of the DCE pattern may be needed.

Sandrasegaran et al. [15], Zhang et al. [19] and Lassel et al. [20] found that the ADC levels were significantly different between benign and malignant renal tumors. However, simply comparing the differences between benign and malignant renal tumors can easily lead to statistical errors due to the distribution of the common types of tumors in the population. Therefore, we classified each major tumor subtype separately using different characteristics so that the results will reflect a more accurate comparison. In our study, ccRCC exhibited a higher ADC level than both AML and pRCC. This result contradicted the conclusions of some previous articles, which suggested that benign renal tumors, including cysts, had a higher ADC value than RCCs [13-15, 19, 20]. In contrast, similar to in our study, Hötker et al. [21] separately measured the ADC values of AML, oncocytoma, ccRCC, pRCC, chRCC, and unclassified RCC and found that ccRCC had the highest ADC level in comparison to the other types of renal tumors. Nonetheless, larger and ideally prospective studies of different benign and malignant subtypes of SRMs are still needed to determine the exact ADC value threshold for differential diagnosis. 
In our study, the ADC values of high-grade ccRCC and any high-grade RCC were significantly lower than those of low-grade ccRCC and any low-grade RCC, indicating that the ADC value is an independent imaging factor associated with tumor grade. Mytsyk et al. [13] observed a difference in the ADC values of different Fuhrman grades of ccRCC, with high-grade tumors presenting lower ADC values and low-grade lesions presenting higher ADC values, which is consistent with our study. High-grade malignant tumors have a higher cell density and stronger angiogenic activity than low-grade malignancies, so water diffusion is more restricted. This phenomenon can be seen not only in ccRCC but also in all RCCs.

In our study, the patients with high-grade ccRCC or any high-grade RCC were significantly older than those with low-grade ccRCC or any low-grade RCC. Mukhopadhyay et al. [22] concluded that patient age is an independent prognostic factor of RCCs. Further clarification of the relationships among patient age, Fuhrman nuclear grade, and tumor stage and their association with prognostic factors may be indicated in the future.

MRI has some disadvantages, such as limited accessibility, slow scanning speed, high cost, and unsuitability for claustrophobic patients. However, overall, MRI is considered a better tool than CT because its multiple parameters can provide more information regarding tumors than simple CT findings. The traditional MRI, DCE, and DWI sequences provide a wide range of opportunities to deeply explore the intrinsic characteristics of tumors.

There are several limitations in our study. First, CT is still the major presurgical imaging tool for SRMs surveyed in our hospital, and only patients considered difficult to diagnose undergo MRI, which resulted in an insufficient number of MRI cases. Second, most of the RCCs were ccRCC in our study, and the number of other subtypes of RCC was relatively small. This may make it difficult to statistically analyze different subtypes. Third, some patients with poor renal function were not imaged with a contrast agent, limiting the data collection of AWI and DCE values.

In conclusion, multiparametric MRI is useful in differentiating among common pathological types of SRMs. Additionally, ADC values may be helpful in evaluating the histological grade of RCC.

Author Contributions All authors contributed to the study conception and design. Material preparation, data collection and data analysis were performed by M-CL and Y-LT. The first draft of the manuscript was written by M-CL. Y-LT and all authors commented on previous versions of the manuscript. All authors have read and approved the final manuscript.

Funding No funding was received to assist with the preparation of this manuscript.
Data Availability Data are not available due to ethical and legal restrictions.Code Availability Not applicable.

\section{Declarations}

Conflict of interest The authors have no conflicts of interest to declare that are relevant to the content of this article.

Ethical Approval This research was conducted retrospectively from data obtained for clinical purposes. The study was reviewed and approved by the IRB of Veterans General Hospital, Approval Number CE21214A.

Informed Consent Informed consent was not required because this study was a retrospective review of patient's images and pathological results, with no risk of harm to patients.

Consent for Publication Not applicable.

Open Access This article is licensed under a Creative Commons Attribution 4.0 International License, which permits use, sharing, adaptation, distribution and reproduction in any medium or format, as long as you give appropriate credit to the original author(s) and the source, provide a link to the Creative Commons licence, and indicate if changes were made. The images or other third party material in this article are included in the article's Creative Commons licence, unless indicated otherwise in a credit line to the material. If material is not included in the article's Creative Commons licence and your intended use is not permitted by statutory regulation or exceeds the permitted use, you will need to obtain permission directly from the copyright holder. To view a copy of this licence, visit http://creativecommons.org/licenses/by/4.0/.

\section{References}

1. Vendrami, C. L., Villavicencio, C. P., DeJulio, T. J., Chatterjee, A., Casalino, D. D., Horowitz, J. M., Oberlin, D. T., Yang, G.-Y., Nikolaidis, P., \& Miller, F. H. (2017). Differentiation of solid renal tumors with multiparametric MR imaging. RadioGraphics, 37(7), 2026-2042. https://doi.org/10.1148/rg.2017170039

2. Sun, M. R., Ngo, L., Genega, E. M., Atkins, M. B., Finn, M. E., Rofsky, N. M., \& Pedrosa, I. (2009). Renal cell carcinoma: Dynamic contrast-enhanced MR imaging for differentiation of tumor subtypes - Correlation with pathologic findings. Radiology, 250(3), 793-802. https://doi.org/10.1148/radiol.2503080995

3. Kay, F. U., Canvasser, N. E., Xi, Y., Pinho, D. F., Costa, D. N., Diaz de Leon, A., Khatri, G., Leyendecker, J. R., Yokoo, T., Lay, A. H., Kavoussi, N., Koseoglu, E., Cadeddu, J. A., \& Pedrosa, I. (2018). Diagnostic performance and interreader agreement of a standardized MR imaging approach in the prediction of small renal mass histology. Radiology, 287(2), 543-553. https://doi.org/ 10.1148/radiol.2018171557

4. Kim, J. K., Kim, S. H., Jang, Y. J., Ahn, H., Kim, C. S., Park, H., Lee, J. W., Kim, S., \& Cho, K. S. (2006). Renal angiomyolipoma with minimal fat: Differentiation from other neoplasms at double-echo chemical shift FLASH MR imaging. Radiology, 239(1), 174-180. https://doi.org/10.1148/radiol.2391050102 
5. Finelli, A., Ismaila, N., Bro, B., Durack, J., Eggener, S., Evans, A., Gill, I., Graham, D., Huang, W., Jewett, M. A. S., Latcha, S., Lowrance, W., Rosner, M., Shayegan, B., Thompson, R. H., Uzzo, R., \& Russo, P. (2017). Management of small renal masses: American Society of Clinical Oncology clinical practice guideline. Journal of Clinical Oncology, 35(6), 668-680. https://doi.org/10.1200/jco. 2016.69.9645

6. Blumenfeld, A. J., Guru, K., Fuchs, G. J., \& Kim, H. L. (2010). Percutaneous biopsy of renal cell carcinoma underestimates nuclear grade. Urology, 76(3), 610-613. https://doi.org/10.1016/j. urology.2009.09.095

7. Oliva, M. R., Glickman, J. N., Zou, K. H., Teo, S. Y., Mortelé, K. J., Rocha, M. S., \& Silverman, S. G. (2009). Renal cell carcinoma: $\mathrm{t} 1$ and $\mathrm{t} 2$ signal intensity characteristics of papillary and clear cell types correlated with pathology. American Journal of Roentgenology, 192(6), 1524-1530. https://doi.org/10.2214/ajr.08.1727

8. Sacco, E., Pinto, F., Totaro, A., D’Addessi, A., Racioppi, M., Gulino, G., Volpe, A., Marangi, F., D’Agostino, D., \& Bassi, P. (2011). Imaging of renal cell carcinoma: State of the art and recent advances. Urology International, 86(2), 125-139. https://doi.org/ $10.1159 / 000322724$

9. Lopes Vendrami, C., Parada Villavicencio, C., DeJulio, T. J., Chatterjee, A., Casalino, D. D., Horowitz, J. M., Oberlin, D. T., Yang, G. Y., Nikolaidis, P., \& Miller, F. H. (2017). Differentiation of solid renal tumors with multiparametric MR imaging. RadioGraphics, 37(7), 2026-2042. https://doi.org/10.1148/rg.20171 70039

10. Hötker, A. M., Mazaheri, Y., Wibmer, A., Karlo, C. A., Zheng, J., Moskowitz, C. S., Tickoo, S. K., Russo, P., Hricak, H., \& Akin, O. (2017). Differentiation of clear cell renal cell carcinoma from other renal cortical tumors by use of a quantitative multiparametric MRI approach. American Journal of Roentgenology, 208(3), W85-W91. https://doi.org/10.2214/AJR.16.16652

11. Johnson, B. A., Kim, S., Steinberg, R. L., de Leon, A. D., Pedrosa, I., \& Cadeddu, J. A. (2019). Diagnostic performance of prospectively assigned clear cell Likelihood Scores (ccLS) in small renal masses at multiparametric magnetic resonance imaging. Urologic Oncology, 37(12), 941-946. https://doi.org/10.1016/j.urolonc. 2019.07.023

12. Lima, F., Elias, J., Chahud, F., Reis, R. B., \& Muglia, V. F. (2020). Diagnostic accuracy of signal loss in in-phase gradient-echo images for differentiation between small renal cell carcinoma and lipid-poor angiomyolipomas. The British Journal of Radiology, 93(1108), 20190975. https://doi.org/10.1259/bjr.20190975

13. Mytsyk, Y., Dutka, I., Borys, Y., Komnatska, I., Shatynska-Mytsyk, I., Farooqi, A. A., Gazdikova, K., Caprnda, M., Rodrigo, L., \& Kruzliak, P. (2017). Renal cell carcinoma: Applicability of the apparent coefficient of the diffusion-weighted estimated by MRI for improving their differential diagnosis, histologic subtyping, and differentiation grade. International Urology and Nephrology, 49(2), 215-224. https://doi.org/10.1007/s11255-016-1460-3

14. Razek, A. A., Farouk, A., Mousa, A., \& Nabil, N. (2011). Role of diffusion-weighted magnetic resonance imaging in characterization of renal tumors. Journal of Computer Assisted Tomography, 35(3), 332-336. https://doi.org/10.1097/RCT.0b013e318219fe76

15. Sandrasegaran, K., Sundaram, C. P., Ramaswamy, R., Akisik, F. M., Rydberg, M. P., Lin, C., \& Aisen, A. M. (2010). Usefulness of diffusion-weighted imaging in the evaluation of renal masses. American Journal of Roentgenology, 194(2), 438-445. https://doi. org/10.2214/ajr.09.3024

16. Roy, C., Sauer, B., Lindner, V., Lang, H., Saussine, C., \& Jacqmin, D. (2007). MR Imaging of papillary renal neoplasms: Potential application for characterization of small renal masses. European Radiology, 17(1), 193-200. https://doi.org/10.1007/ s00330-006-0271-9

17. Pedrosa, I., Sun, M. R., Spencer, M., Genega, E. M., Olumi, A. F., Dewolf, W. C., \& Rofsky, N. M. (2008). MR imaging of renal masses: Correlation with findings at surgery and pathologic analysis. RadioGraphics, 28(4), 985-1003. https://doi.org/10.1148/rg. 284065018

18. Cornelis, F., Tricaud, E., Lasserre, A. S., Petitpierre, F., Bernhard, J. C., Le Bras, Y., Yacoub, M., Bouzgarrou, M., Ravaud, A., \& Grenier, N. (2014). Routinely performed multiparametric magnetic resonance imaging helps to differentiate common subtypes of renal tumours. European Radiology, 24(5), 1068-1080. https:// doi.org/10.1007/s00330-014-3107-z

19. Zhang, H., Gan, Q., Wu, Y., Liu, R., Liu, X., Huang, Z., Yuan, F., Kuang, M., \& Song, B. (2016). Diagnostic performance of diffusion-weighted magnetic resonance imaging in differentiating human renal lesions (benignity or malignancy): A meta-analysis. Abdominal Radiology (NY), 41(10), 1997-2010. https://doi.org/ 10.1007/s00261-016-0790-z

20. Lassel, E. A., Rao, R., Schwenke, C., Schoenberg, S. O., \& Michaely, H. J. (2014). Diffusion-weighted imaging of focal renal lesions: A meta-analysis. European Radiology, 24(1), 241-249. https://doi.org/10.1007/s00330-013-3004-x

21. Hötker, A. M., Mazaheri, Y., Wibmer, A., Zheng, J., Moskowitz, C. S., Tickoo, S. K., Russo, P., Hricak, H., \& Akin, O. (2016). Use of DWI in the differentiation of renal cortical tumors. American Journal of Roentgenology, 206(1), 100-105. https://doi.org/10. 2214/ajr.14.13923

22. Mukhopadhyay, S. G., Mukherjee, K., \& Kr Manna, A. (2015). Renal tumours in adults with correlation between Fuhrman grading and proliferative marker. Iranian Journal of Pathology, 10(4), 281-289.

\section{Authors and Affiliations}

\section{Ming-Cheng Liu ${ }^{1,2} \cdot$ Yi-Jui Liu ${ }^{2,3} \cdot$ Yen-Ting Lin ${ }^{1,4} \cdot$ Siu-Wan Hung ${ }^{1,5,6} \cdot$ Jyh-Wen Chai $^{1} \cdot$ Si-Wa Chan ${ }^{7,8,9}$. Kun-Yuan Chiu ${ }^{10,11}$. Chih-Hao Chang ${ }^{12} \cdot$ Ya-Ling Tsou ${ }^{1,13}$ (B)}

Ming-Cheng Liu

penguin2046@gmail.com

Yi-Jui Liu

erliu@fcu.edu.tw

Yen-Ting Lin

ymerically@gmail.com

Siu-Wan Hung

swhtcvgh@gmail.com
Jyh-Wen Chai

hubt@vghtc.gov.tw

Si-Wa Chan

chan.siwa@gmail.com

Kun-Yuan Chiu

kychiu37782002@gmail.com

Chih-Hao Chang

chang.chih.hao@outlook.com 
1 Department of Radiology, Taichung Veterans General Hospital, Taichung, Taiwan

2 Ph.D. Program of Electrical and Communications Engineering, Feng Chia University, Taichung, Taiwan

3 Department of Automatic Control Engineering, Feng Chia University, Taichung, Taiwan

4 Institute of Clinical Medicine, National Yang-Ming University, Taipei, Taiwan

5 Department of Veterinary Medicine, National Chung Hsing University, Taichung, Taiwan

6 School of Medical Imaging and Radiological Sciences, Chung Shan Medical University, Taichung, Taiwan

7 Department of Medical Imaging, Taichung Tzu Chi Hospital, Buddhist Tzu Chi Medical Foundation, Taichung, Taiwan
8 School of Medicine, Tzu Chi University, Hualien, Taiwan

9 Department of Medical Imaging Radiological Science, Central Taiwan University of Science and Technology, Taichung, Taiwan

10 Division of Urology, Department of Surgery, Taichung Veterans General Hospital, Taichung, Taiwan

11 Department of Applied Chemistry, National Chi Nan University, Nantou, Taiwan

12 Nagoya University, Nagoya, Japan

13 Department of Radiology, Taichung Veterans General Hospital, No. 1650, Sec. 4, Taiwan Boulevard, Taichung 40705, Taiwan 